\title{
Generating Functional for the $S$-Matrix in Liouville Theory
}

\section{George Jorjadze $\mathrm{e}^{a, b, c, *}$ and Stefan Theisen ${ }^{c}$}

${ }^{a}$ Free University of Tbilisi, Agmashenebeli Alley 240, 0159, Tbilisi, Georgia

${ }^{b}$ Razmadze Mathematical Institute of TSU,

Tamarashvili 6, 0177, Tbilisi, Georgia

${ }^{c}$ Max-Planck-Institut für Gravitationsphysik, Albert-Einstein-Institut, 14476, Golm, Germany

E-mail: g.jorjadze@freeuni.edu.ge, theisen@aei.mpg.de

We recently proposed a functional integral representation for the generating functional of $S$-matrix elements of Liouville theory on a cylinder. The functional integral is defined in terms of a nonlocal one-dimensional action on a circle. We review and elaborate on this proposal and subject it to non-perturbative checks.

RDP workshop 'Recent Advances in Mathematical Physics' - Regio2020

online

December 5-6, 2020

${ }^{*}$ Speaker 
Liouville theory is an interesting interacting two-dimensional quantum field theory, which has appeared and has been studied in various contexts. Being a conformal and integrable field theory it certainly has many special properties not shared by generic two- and higher-dimensional QFT's, but nevertheless it is a highly non-trivial Lagrangian field theory which deserves further study. For background and a guide to the large literature we refer to the reviews [1-3].

In this contribution we elaborate on and further study the approach to the scattering matrix in Liouville theory on a cylinder which we presented in [4]. But before going into technical details, we outline the general idea and strategy.

As a consequence of integrability and conformal invariance, in each chiral sector the relation between in- and out-fields is known. This can be turned into an exact equation for the quantum $S$-matrix, which is the collection of all matrix elements between asymptotic in- and out-states. It is most conveniently presented as the generating functional $\mathcal{S}$ for these matrix elements, defined by coherent states, and as such depends on external sources $j$. The exact matrix elements, which are the coefficients in the expansion of $\mathcal{S}$ in powers of the sources, can be obtained by solving this equation with a power series Ansatz. In practice this is tedious but easily done for few and small mode numbers of the scattered states.

If we write $\mathcal{S}=e^{\frac{i}{\hbar} F_{q}}$, the equation for $\mathcal{S}$ turns into an equation for $F_{q}$. In this equation we can perform the semi-classical limit by setting $\hbar=0$, thus obtaining an equation for the generating functional of classical scattering amplitudes, $F_{c l}$, which is straightforwardly solved order by order in the sources. Having the generating function for classical amplitudes, a natural question is whether they can be associated with tree-level Feynman diagrams derived from some classical field theory, specified by an action $S$, the Legendre transform of $F_{c l}$. Indeed, the equation satisfied by $F_{c l}$ or, alternatively, directly the relation between the asymptotic in- and out-fields, can be used to find $S$. This is a non-local action for a scalar field on a circle and not the original Liouville action which is local and defined on a cylinder, but it has some resemblance with it. The classical scattering amplitudes can then be computed by summing all connected tree-level Feynman diagrams with a fixed number of external legs.

The next obvious question is whether the system specified by the action $S$ can be quantized and whether the quantum amplitudes agree with the scattering matrix of Liouville theory, which are in principle known (see the discussion above). We claim that this is indeed the case and give the functional integral representation for the quantum amplitudes which uses the action $S$. We have not succeeded in proving this claim but we present convincing perturbative and non-perturbative evidence by comparing the correlation functions computed from the field theory defined by $S$ with the exact $\mathcal{S}$-matrix elements.

In the following we collect the relevant features of Liouville theory and go through all the steps which are outlined above in some detail, which lead to the functional integral representation of the $S$-matrix. As an extensive perturbative analysis was already presented in [4], in this contribution we will mainly concentrate on non-perturbative checks.

We now state known results about Liouville theory which are used later on. Further details can be found in the cited reviews and in [4]. The Liouville action is

$$
S=\frac{1}{2} \int_{\Sigma} d^{2} \sigma\left(\partial_{\mu} \Phi \partial^{\mu} \Phi-4 \mu^{2} e^{2 \Phi}\right)
$$


$\mu$ is a positive constant and $\Sigma$ is the infinite cylinder with coordinates $\sigma \in[0,2 \pi)$ and $\tau \in(-\infty,+\infty)$. The general solution can be written in the form

$$
e^{-\Phi}=e^{-\Phi_{\text {in }}}+e^{-\Phi_{\text {out }}}
$$

where $\left(\sigma^{ \pm}=\tau \pm \sigma\right)$

$$
\Phi_{\text {in }}=\phi_{\text {in }}\left(\sigma^{+}\right)+\bar{\phi}_{\text {in }}\left(\sigma^{-}\right), \quad \Phi_{\text {out }}=\phi_{\text {out }}\left(\sigma^{+}\right)+\bar{\phi}_{\text {out }}\left(\sigma^{-}\right)
$$

Note that $\Phi_{\text {in/out }}$, being sums of left and right-moving parts, satisfy the free field equation. They can be expanded as

$$
\Phi_{\text {in }}=q_{\text {out }} \pm p \tau+\text { oscillators }
$$

with $p>0$ and, therefore, $\Phi \rightarrow \Phi_{\text {int }}$ as $\tau \rightarrow \mp \infty$. Thus $\Phi_{\text {in }}$ aut are identified as the free asymptotic inand out-fields.

From now on we will mainly look at the left-movers which, except in the zero-mode sector, completely decouple from the right-movers. They have the mode expansion

$$
\phi_{\text {in }}\left(\sigma^{+}\right)=q+\frac{1}{2} p \sigma^{+}+i \sum_{n \neq 0} \frac{a_{n}}{n} e^{-i n \sigma^{+}}, \quad \phi_{\text {out }}\left(\sigma^{+}\right)=\tilde{q}+\frac{1}{2} \tilde{p} \sigma^{+}+i \sum_{n \neq 0} \frac{b_{n}}{n} e^{-i n \sigma^{+}}
$$

with $\tilde{p}=-p$. One can show that the in- and out-fields are related via

$$
\phi_{\text {in }}^{\prime}-\phi_{\text {out }}^{\prime}=\mu e^{\phi_{\text {in }}+\phi_{\text {out }}}
$$

where the prime denotes derivative w.r.t. the argument, i.e. $\sigma^{+}$. This equation defines a canonical transformation between in- and out-fields and contains the full dynamical content of classical scattering of. The reason for such a simple relation to exist is the classical integrability of the theory and the fact that this relation exists for each chiral sector is a consequence of conformal invariance.

Following [4], we choose the positive modes of $\phi_{\text {in }}$ and the negative modes of $\phi_{\text {out }}$ and the momentum $p$ as independent variables and introduce the generating function of the canonical transformation between in- and out-fields of the type $F\left(p, b_{m}^{*}, a_{m}\right)$ with $m>0$ and $b_{m}^{*}=b_{-m}$ due to the reality of $\phi$. The other half of the canonical variables are obtained as

$$
q+\tilde{q}=\frac{\partial F}{\partial p}, \quad a_{m}^{*}=\frac{i m}{2} \frac{\partial F}{\partial a_{m}}, \quad b_{m}=\frac{i m}{2} \frac{\partial F}{\partial b_{m}^{*}}
$$

Upon quantization the Fourier modes become operators and satisfy the canonical commutation relations $(m>0)$

$$
[\hat{q}, \hat{p}]=i \hbar, \quad\left[\hat{a}_{m}, \hat{a}_{m}^{\dagger}\right]=\frac{1}{2} \hbar m, \quad[\hat{\tilde{q}}, \hat{\tilde{p}}]=i \hbar, \quad\left[\hat{b}_{m}, b_{m}^{\dagger}\right]=\frac{1}{2} \hbar m
$$

The $p$-dependent $(p>0)$ vacuum state for the chiral in-field is defined by the conditions

$$
\hat{p}|p, 0\rangle_{\text {in }}=p|p, 0\rangle_{\text {in }}, \quad \hat{a}_{m}|p, 0\rangle_{\text {in }}=0, \quad \text { for } \quad m>0
$$

and coherent states are constructed as

$$
|p, a\rangle=\exp \left(\frac{2}{\hbar} \sum_{m>0} \frac{1}{m} a_{m} \hat{a}_{m}^{\dagger}\right)|p, 0\rangle_{\mathrm{in}}
$$


They span the Hilbert space of the chiral sector of Liouville theory. The out-field operators and bra-vectors $\left\langle b^{*}, \tilde{p}\right|$, with $\tilde{p}<0$, are defined similarly and one finds

$$
\begin{array}{ll}
\hat{a}_{m}|p, a\rangle=a_{m}|p, a\rangle, & \hat{a}_{m}^{\dagger}|p, a\rangle=\frac{1}{2} \hbar m \frac{\partial}{\partial a_{m}}|p, a\rangle \\
\left\langle b^{*}, \tilde{p}\right| \hat{b}_{m}^{\dagger}=b_{m}^{*}\left\langle b^{*}, \tilde{p}\right|, & \left\langle b^{*}, \tilde{p}\right| \hat{b}_{m}=\frac{1}{2} \hbar m \frac{\partial}{\partial b_{m}^{*}}\left\langle b^{*}, \tilde{p}\right|
\end{array}
$$

Our aim is to compute $S$-matrix elements $\left\langle b^{*}, \tilde{p} \mid p, a\right\rangle$. In a first step we insert the canonical operators and find

$$
\left\langle b^{*}, \tilde{p}|\hat{\tilde{p}}+\hat{p}| p, a\right\rangle=(\tilde{p}+p)\left\langle b^{*}, \tilde{p} \mid p, a\right\rangle, \quad\left\langle b^{*}, \tilde{p}|\hat{\tilde{q}}+\hat{q}| p, a\right\rangle=i \hbar\left(\partial_{\tilde{p}}-\partial_{p}\right)\left\langle b^{*}, \tilde{p} \mid p, a\right\rangle
$$

If we require $\hat{\tilde{p}}=-\hat{p}$, which we know to be satisfied by the classical solutions, the 1.h.s. of the first equation vanishes and yields

$$
\left\langle b^{*}, \tilde{p} \mid p, a\right\rangle=\mathcal{S}\left(p, b^{*}, a\right) \delta(p+\tilde{p})
$$

If we assume that the classical relations (7) are valid at the quantum level, we obtain from (11)-(13)

$$
-i \hbar \frac{\partial \mathcal{S}}{\partial p}=\frac{\partial F}{\partial p} \mathcal{S}, \quad-i \hbar \frac{\partial \mathcal{S}}{\partial a_{m}}=\frac{\partial F}{\partial a_{m}} \mathcal{S}, \quad-i \hbar \frac{\partial \mathcal{S}}{\partial b_{m}^{*}}=\frac{\partial F}{\partial b_{m}^{*}} \mathcal{S}
$$

and find

$$
\mathcal{S}\left(p, b^{*}, a\right)=e^{\frac{i}{\hbar} F\left(p, b^{*}, a\right)}
$$

This semi-classical result is valid up to $O(\hbar)$ corrections, as in its derivation we ignored the issue of operator ordering.

If we are interested in the semi-classical scattering matrix it suffices to determine $F$. This can be done with the help of (6) and (7). For instance, for the zero modes this leads to the relation $q+\tilde{q}=\log (p / \mu)+$ oscillators which integrates to

$$
F=p[\log (p / \mu)-1]+\text { oscillators }
$$

One can, in principle, continue and work out the oscillator contributions, but it becomes quickly very tedious. A more economical, but still tedious way is to take the derivative of (6). This leads to

$$
T(x)=\phi_{\text {in }}^{\prime 2}(x)-\phi_{\text {in }}^{\prime \prime}(x)=\phi_{\text {out }}^{\prime 2}(x)-\phi_{\text {out }}^{\prime \prime}(x)
$$

which shows that the 'improved' free-field stress tensors of the in- and out-fields coincide. If we expand $T$ into modes,

$$
T(x)=\sum_{n \in \mathbb{Z}} L_{n} e^{-i n x}
$$

and express the $L_{n}^{\text {in }}$ and $L_{n}^{\text {out }}$ in terms of modes of $\phi_{\text {in }}$ and $\phi_{\text {out }}$, respectively, replace half of them via the relations (7) and equate $L_{n}^{\text {in }}=L_{n}^{\text {out }}$, we get a system of coupled non-linear inhomogeneous differential equations for $F$. They can be solved as a power series in the independent variables $a_{m}, b_{m}^{*}$ with $m>0$. The coefficients are functions of $p$. Details can be found in [4]. Here we go directly to the quantum version of this equation, from which the equation for the semiclassical $F$ can be obtained in the limit $\hbar \rightarrow 0$. 
It is well known that a consistent quantization of Liouville theory leads to a deformation of the improvement term in the stress tensor [5,6]. This is required in order for the potential term in the Liouville action to be a marginal deformation, thereby preserving conformal invariance and it introduces a quantum contribution to the central charge. The quantum Virasoro generators, i.e. the modes of $T=: \phi^{\prime 2}:+(1+\hbar) \phi^{\prime \prime}$ in terms of the in-field variables are

$$
\begin{aligned}
& \hat{L}_{0}=\frac{1}{4} p^{2}+2 \sum_{j \geq 1} \hat{a}_{-j} \hat{a}_{j} \\
& \hat{L}_{m}=(\hat{p}+i m \eta) \hat{a}_{m}+\sum_{i, j \neq 0} \hat{a}_{i} \hat{a}_{j} \delta_{i+j, m}
\end{aligned}
$$

$\eta=1+\hbar$ and, as always, $\hat{a}_{m}^{\dagger}=a_{-m}$ and no ordering is required in the second line. The same generators in terms of the out-field variables are obtained by the replacements $p \mapsto-p, \hat{a}_{j} \mapsto \hat{b}_{j}$.

The zero mode operators fix the transition amplitude between the in- and out-coherent states as in (13), where the freedom is given by the function $\mathcal{S}\left(p, b^{*}, a\right)$. Inserting the Virasoro generators between the coherent states, $\left\langle\tilde{p}, b^{*}\left|\hat{L}_{n}\right| p, a\right\rangle$, and using the relations (11), one obtains equations for $\mathcal{S}\left(p, b^{*}, a\right)$. At this point it is convenient to introduce a covariant notation for the independent variables and to define

$$
-\frac{i}{2} \epsilon(n) j_{n}=\left\{\begin{array}{ll}
a_{n} & n>0 \\
b_{n} & n<0
\end{array} \quad \epsilon(k)=\operatorname{sign}(k)=\frac{k}{|k|}\right.
$$

In terms of those we find the following equations for $\mathcal{S}(p, j)$ :

$$
\begin{gathered}
\sum_{k \neq 0} k j_{k} \frac{\partial \mathcal{S}}{\partial j_{k}}=0 \\
2 \hbar n(|n| \eta+i p) \frac{\partial \mathcal{S}}{\partial j_{n}}-\epsilon(n)(|n| \eta-i p) j_{-n} \mathcal{S}+\hbar \sum_{k, l \neq 0}[\epsilon(k)-\epsilon(l)]|l| j_{k} \frac{\partial \mathcal{S}}{\partial j_{-l}} \delta_{k+l+n} \\
+\sum_{k, l \neq 0}[\epsilon(k)+\epsilon(l)]\left(-\frac{1}{4} j_{k} j_{l} \mathcal{S}+\hbar^{2} k l \frac{\partial^{2} \mathcal{S}}{\partial j_{-k} \partial j_{-l}}\right) \delta_{k+l+n}=0
\end{gathered}
$$

The first one is a homogeneity condition while the second is valid for $n \neq 0$. This equation simplifies if we define

$$
\mathcal{S}=e^{-\frac{1}{4 \hbar} \sum_{n \neq 0} \frac{j n j-n}{|n|}} \tilde{\mathcal{S}}
$$

It becomes

$-n \eta j_{-n} \tilde{\mathcal{S}}+\hbar n(|n| \eta+i p) \frac{\partial \tilde{\mathcal{S}}}{\partial j_{n}}-\hbar \sum_{k, l \neq 0} l j_{k} \frac{\partial \tilde{\mathcal{S}}}{\partial j_{-l}} \delta_{k+l+n}+\frac{1}{2} \hbar^{2} \sum_{k, l \neq 0}[\epsilon(k)+\epsilon(l)] k l \frac{\partial^{2} \tilde{\mathcal{S}}}{\partial j_{-k} \partial j_{-l}} \delta_{k+l+n}=0$

As the following analysis will show, the redefinition (23) is crucial.

We represent $\mathcal{S}$ (and $\tilde{\mathcal{S}}$ ) in the form ${ }^{1}$

$$
\mathcal{S}=R(p) e^{\frac{i}{\hbar} F_{q}}, \quad\left(\tilde{\mathcal{S}}=R(p) e^{-\frac{1}{\hbar} \tilde{F}_{q}}\right)
$$

\footnotetext{
${ }^{1}$ If one includes both chiral sectors, this becomes $\mathcal{S}=R(p) e^{\frac{i}{\hbar}\left(F_{q}+\bar{F}_{q}\right)} . F_{q}$ starts at $O\left(j^{2}\right)$. In the classical case, considered below, we include in $F$ also the zero-mode part, as in (16).
} 
where $R(p)$, the reflection amplitude, is known as the 2-point function of Liouville theory. It cancels in the above equations for $\mathcal{S}$ and was computed by other means in [7, 8].

The Ansatz (25) turns the linear equation for $\tilde{\mathcal{S}}$ to the following non-linear equation for $\tilde{F}_{q}$ :

$$
\begin{aligned}
i n(|n| \eta+i p) \frac{\partial \tilde{F}_{q}}{\partial j_{n}}- & n \eta j_{-n}-i \sum_{k, l \neq 0} l j_{k} \frac{\partial \tilde{F}_{q}}{\partial j_{-l}} \delta_{k+l+n} \\
& -\frac{1}{2} \sum_{k, l \neq 0}[\epsilon(k)+\epsilon(l)] k l\left(\frac{\partial \tilde{F}_{q}}{\partial j_{-k}} \frac{\partial \tilde{F}_{q}}{\partial j_{-l}}-i \hbar \frac{\partial^{2} \tilde{F}_{q}}{\partial j_{-k} \partial j_{-l}}\right) \delta_{k+l+n}=0
\end{aligned}
$$

In the limit $\hbar \rightarrow 0$ the last term is absent, $\eta \rightarrow 1$ and we obtain an equation for the semi-classical scattering amplitude:

$$
\operatorname{in}(|n|+i p) \frac{\partial \tilde{F}}{\partial j_{n}}-n j_{-n}-i \sum_{k, l \neq 0} l j_{k} \frac{\partial \tilde{F}}{\partial j_{-l}} \delta_{k+l+n}-\sum_{k, l \neq 0}|k| l \frac{\partial \tilde{F}}{\partial j_{-k}} \frac{\partial \tilde{F}}{\partial j_{-l}} \delta_{k+l+n}=0
$$

This is the equation which we would have obtained had we followed the steps outlined immediately below eq.(18). Our task is to solve (24).

A straightforward way to do this is to make a power series Ansatz (in $j$ ) for $\tilde{\mathcal{S}}$, insert it into (24) and solve it order by order. This is tedious but can be done e.g. with the help of Mathematica. A more interesting way to proceed was already outlined in the introduction and goes as follows: we start with the classical relation (6) which we write in terms of the oscillator modes:

$$
p+\sum_{n \neq 0} \beta_{n} e^{-i n x}=\mu e^{q_{+}} e^{i \sum_{n \neq 0} \frac{1}{n} \gamma_{n} e^{-i n x}}
$$

where we have defined $q_{+}=q+\tilde{q}$ and

$$
\gamma_{n}=a_{n}+b_{n}, \quad \beta_{n}=a_{n}-b_{n}
$$

They are related to $j_{n}$ given in (20) via

$$
j_{n}=i \epsilon(n) \gamma_{n}+i \beta_{n}
$$

In analogy to the definition of $\tilde{F}_{q}$, we define the classical $\tilde{F}$ as (cf. footnote 1 )

$$
\tilde{F}=F-p\left(\ln \frac{p}{\mu}-1\right)-2 i \sum_{m>0} \frac{1}{m} b_{m}^{*} a_{m}
$$

and using (7) we find

$$
\frac{\partial \tilde{F}}{\partial j_{-n}}=\frac{1}{n} \gamma_{n} \equiv-i \varphi_{n}
$$

Note that while the $j_{n}$ did not satisfy any reality condition, $\varphi_{n}^{*}=\varphi_{-n}$, i.e. they are the non-zero Fourier modes of the real field $\phi_{\text {in }}+\phi_{\text {out }}$. We define $\check{\varphi}(x)=\sum_{k \neq 0} \varphi_{k} e^{-i k x}$ and reserve $\varphi(x)$ for a generic scalar field on the circle, which also includes a zero mode.

Using these definitions and (28), we can write (30) in the form

$$
j_{n}=|n| \varphi_{n}+i \mu e^{q_{+}} \int_{0}^{2 \pi} \frac{d x}{2 \pi} e^{i n x} e^{\check{\varphi}(x)} \stackrel{!}{=} \frac{\partial S}{\partial \varphi_{-n}}
$$


where $S$ is defined to be the Legendre transform of $\tilde{F}$.

If we use the zero mode of eq. (28) to express $q_{+}$through $\varphi_{k}$, keeping $p$ fixed, we can integrate (33) and find

$$
S=\frac{1}{2} \sum \varphi_{n}|n| \varphi_{-n}+i p \ln U(\check{\varphi}) \quad \text { with } \quad U(\check{\varphi})=\int_{0}^{2 \pi} \frac{d x}{2 \pi} e^{\check{\varphi}(x)}
$$

$S$ is the classical action whose Legendre transform is the generating functional for classical, i.e. tree-level scattering amplitudes. Up to an irrelevant additive constant, it is completely determined by the classical integrability of Liouville theory, expressed via (6).

The classical action $S$ is non-local and, in particular, the non-local potential makes a perturbative treatment rather cumbersome. We can arrive at a local potential if we 'integrate in' an additional variable which will play the role of the zero-mode $\varphi_{0}$. Indeed, it is readily verified that the following action is completely equivalent (and hence we use the same symbol $S$ )

$$
\begin{aligned}
S & =\frac{1}{2} \sum \varphi_{n}|n| \varphi_{-n}+i p \int_{0}^{2 \pi} \frac{d x}{2 \pi} e^{\varphi(x)}-i p\left(\varphi_{0}+1\right) \\
& =\frac{1}{2} \sum_{n \in \mathbb{Z}} \varphi_{n}(|n|+i p) \varphi_{-n}+i p \int_{0}^{2 \pi} \frac{d x}{2 \pi} \sum_{r \geq 3} \frac{1}{r !} \varphi^{r}(x)
\end{aligned}
$$

where $\varphi(x)$ also includes a zero mode. As the equation of motion for $\varphi_{0}$ is algebraic, we can use it to eliminate $\varphi_{0}$ and arrive at the original action (34). We will use both forms in the following.

Before proceeding, we will sketch an alternative way of arriving at $S$. If we start from (27) and use (32) and (33), we obtain an equation for $S$ :

$$
n(|n|+i p) \varphi_{-n}-n \frac{\partial S}{\partial \varphi_{n}}-\sum_{k, l \neq 0} l \varphi_{l} \frac{\partial S}{\partial \varphi_{-k}} \delta_{k+l+n}+\sum_{k, l \neq 0}|k| l \varphi_{k} \varphi_{l} \delta_{k+l+n}=0
$$

This equation can be further simplified with the Ansatz

$$
S=\frac{1}{2} \sum_{n \neq 0} \varphi_{n}|n| \varphi_{-n}+V(\check{\varphi})
$$

leading to

$$
-i p n \varphi_{-n}+n \frac{\partial V}{\partial \varphi_{n}}+\sum_{k, l \neq 0} l \varphi_{l} \frac{\partial V}{\partial \varphi_{-k}} \delta_{k+l+n}=0
$$

One verifies that $V=i p \ln U$ is a solution, thus recovering (34).

$\tilde{F}$ contains all the information about the semi-classical scattering amplitudes. If we expand it as

$$
\tilde{F}=\sum_{v=2}^{\infty} \frac{1}{v !} \sum_{n_{i} \neq 0} \tilde{f}_{n_{1}, \ldots, n_{v}}^{(v)} j_{n_{1}} \cdots j_{n_{\nu}} \delta_{n_{1}+\cdots+n_{v}}
$$

then the $\tilde{f}_{n_{1} \ldots n_{\nu}}^{(\nu)}$ are the amplitudes which involve $v$ external states, where those with $n_{i}>0$ are incoming and those with $n_{j}<0$ are outgoing.

From the above discussion it follows

$$
e^{\frac{i}{\hbar} \tilde{F}(j)}=\left.\int D \varphi e^{-\frac{1}{\hbar}(S(\varphi)-\langle\varphi j\rangle)}\right|_{\frac{\delta S}{\delta \varphi}=j}
$$


where

$$
\langle\varphi j\rangle=\int_{0}^{2 \pi} \frac{d x}{2 \pi} j(x) \varphi(x)=\sum_{n \in \mathbb{Z}} j_{n} \varphi_{-n}
$$

The functional integral over $\varphi$ is evaluated at the saddle point which restricts it to connected treelevel amplitudes. Here either form of the action can be used. As written here $S$ contains the zero mode of $\varphi$ and $S$ is given by (35); this leads to simple Feynman rules, as discussed below. $j$ is the external source for the quantum field $\varphi$.

One can now check, and this was done in considerable detail in [4], that the solutions for $\tilde{f}^{(v)}$, which one obtains by inserting the Ansatz (39) into (27), are reproduced as tree-level amplitudes derived from the above functional integral via the Feynman rules, which follow directly from the form of the classical action $S$ :

1. Draw all connected $v$-point tree diagrams with $r$-valent vertices for all $r \geq 3$. Each external line carries an index $n_{i}, i=1, \ldots, v$. The labels of the internal lines are dictated by 'momentum' conservation at each vertex.

2. To each $r$-valent vertex assign a factor $-i p$.

3. To each line, including the external ones, assign a propagator $\frac{1}{|n|+i p}$ where $n$ is the "momentum' carried by the line. All external lines are ingoing.

4. Sum over all distinct diagrams.

While it is a priori clear that this must work for the classical scattering amplitudes, it is by far not obvious that the above functional integral, beyond the saddle point approximation, computes the quantum amplitudes, i.e. the solutions of (24) or (26). We have no general prove that this is indeed true. In [4] we computed a few exact two, three and four point amplitudes by solving (26), and checked that at the lowest non-trivial order in their expansion in $\hbar$ they agree with the one-loop evaluation of the above functional integral, using the Feynman rules. It turns out that there are divergent diagrams which arise from self-contractions of lines emanating from the same vertex, but one can show that for each amplitude they cancel after summing all contributing diagrams. ${ }^{2}$

We will not repeat these computations here but rather turn to our attempts at an exact evaluation of the functional integral. For this it is more convenient to use the form of the classical action $S$ where the zero mode is not included. We therefore want to compute the functional integral

$$
Z[j]=\int \prod_{n \neq 0} d \varphi_{n} \exp \left\{-\frac{1}{\hbar}\left(\frac{1}{2} \sum_{n \neq 0} \varphi_{n}|n| \varphi_{-n}+i p \ln \int \frac{d x}{2 \pi} e^{\check{\varphi}(x)}-\sum_{n \neq 0} \varphi_{n} j_{-n}\right)\right\}
$$

One can verify that, up to a normalization, this is what one obtains upon integrating out $\varphi_{0}$ in (40). The claim is that the reduced $S$-matrix is

$$
\tilde{\mathcal{S}}[j]=\frac{Z[j]}{Z[0]}=e^{\frac{i}{\hbar} \tilde{F}[j]}
$$

\footnotetext{
${ }^{2}$ This was not realized in [4], where a 'normal ordering' prescription was proposed. But this is, after all, not necessary.
} 
It is convenient to define $\hbar=b^{2}$ and to rescale the field and source according to $\varphi_{n} \rightarrow b \varphi_{n}, j_{n} \rightarrow$ $b j_{n}$. Up to an irrelevant normalization, we obtain

$$
Z[j]=\int \prod d \varphi_{n} \exp \left\{-\frac{1}{2} \sum \varphi_{n}|n| \varphi_{-n}-\frac{i p}{b^{2}} \ln \int \frac{d x}{2 \pi} e^{b \check{\varphi}(x)}+\sum \varphi_{n} j_{-n}\right\}
$$

Here and below the sums and produtcs over the mode numbers never include zero modes. They are simply absent. To proceed we choose special discrete values for the momentum $p=i N \hbar$ with $N$ a positive integer. In this case we can write $Z[j]$ in the form

$$
\begin{aligned}
Z[j] & =\int \prod d \varphi_{n} e^{-\frac{1}{2} \sum \varphi_{n}|n| \varphi_{-n}+\sum \varphi_{n} j_{-n}}\left(\int \frac{d x}{2 \pi} e^{b \check{\varphi}}\right)^{N} \\
& =\int \prod_{\alpha=1}^{N} \frac{d x_{\alpha}}{2 \pi} \int \prod d \varphi_{n} \exp \left\{-\frac{1}{2} \sum \varphi_{n}|n| \varphi_{-n}+\sum J_{n} \varphi_{-n}\right\}
\end{aligned}
$$

Here we have defined

$$
J_{n}=j_{n}+b \sum_{\alpha=1}^{N} e^{i n x_{\alpha}}
$$

We can now do the integral over the $\varphi_{n}$ and obtain

$$
Z[j]=e^{\frac{1}{2} \sum j_{n} \frac{1}{|n|} j_{-n}} \int \prod_{\alpha=1}^{N} \frac{d x_{\alpha}}{2 \pi} \exp \left\{b \sum j_{n} \frac{1}{|n|} \sum_{\alpha} e^{-i n x_{\alpha}}+\frac{1}{2} b^{2} \sum_{n} \frac{1}{|n|} \sum_{\alpha, \beta} e^{i n\left(x_{\alpha}-x_{\beta}\right)}\right\}
$$

A $j$-independent normalization factor will cancel against the same factor in $Z[0]$ and was dropped. The second sum in the exponent diverges for $x_{\alpha}=x_{\beta}$. This divergence is independent of the sources $j_{n}$ and also cancels when dividing by $Z[0] .{ }^{3}$ We will also drop these diagonal terms henceforth and restrict the sum to $\alpha \neq \beta$.

For the choice $N=1$, which we consider first, this sum is absent and, after introducing $z_{\alpha}=e^{i x_{\alpha}}$, we obtain

$$
\tilde{S}[j]=e^{\frac{1}{2} \sum j_{n} \frac{1}{|n|} j_{-n}} \oint \frac{d z}{2 \pi i z} e^{b \sum \frac{j_{n}}{|n|} z^{-n}}
$$

This can be evaluated to any order in $j$. For instance, to $O\left(j^{3}\right)$, we find

$$
\begin{aligned}
\tilde{S}[j] & =1+\frac{1}{2 \hbar} \sum_{n \neq 0} \frac{|n|+\hbar}{|n|^{2}} j_{n} j_{-n}+\frac{1}{3 ! \hbar} \sum_{n, m \neq 0} \frac{\hbar}{|n||m||n+m|} j_{n} j_{m} j_{-n-m}+O\left(j^{4}\right) \\
& =1+\frac{1}{\hbar}\left(\frac{1}{2} \sum_{n} \tilde{f}_{-n, n}^{(2)} j_{n} j_{-n}+\frac{1}{3 !} \sum_{n_{1} n_{2} n_{3}} \tilde{f}_{n_{1} n_{2} n_{3}}^{(3)} j_{n_{1}} j_{n_{2}} j_{n_{3}} \delta_{n_{1}+n_{2}+n_{3}}+\ldots\right)
\end{aligned}
$$

where we have undone the rescaling of $j_{n}$. This agrees with the exact expressions for $\tilde{f}^{(2)}$ and $\tilde{f}^{(3)}$ if we evaluate them at $p=i \hbar$. It is due to this special value for $p$ that the $\hbar$ dependence appears so simple. We will see below that this is not generally so and is also clear from the exact expressions, valid for any $p$, which are given in [4] and those previously calculated in [8] by other means.

\footnotetext{
${ }^{3}$ The appearance of the divergence is a consequence of the special choice for $p$. As long as $p$ is real, the functional integral is finite.
} 
We now evaluate $Z[j]$ for $N=2$, i.e. for $p=2 i \hbar$. After introducing complex variables $z_{1,2}$ we find (recall $\left.b^{2}=\hbar\right)$

$$
Z[j]=e^{\frac{1}{2} \sum j_{n} \frac{1}{|n|} j_{-n}} \oint \frac{d z_{1}}{2 \pi i z_{1}} \oint \frac{d z_{2}}{2 \pi i z_{2}} e^{b \sum \frac{j_{n}}{|n|}\left(z_{1}^{-n}+z_{2}^{-n}\right)}\left(\frac{\left(z_{1}-z_{2}\right)^{2}}{z_{1} z_{2}}\right)^{-b^{2}}
$$

To evaluate this we use a result from [9], based on [10], which can be written in the form

$$
\begin{aligned}
& I_{N}(\alpha, \gamma, \beta) \equiv \oint \frac{d z_{1}}{2 \pi i z_{1}} \cdots \frac{d z_{N}}{2 \pi i z_{N}} \prod_{j=1}^{N}\left(1-z_{j}\right)^{\alpha} z_{j}^{\gamma} \prod_{i<j}^{N}\left(z_{i}-z_{j}\right)^{\beta} \\
& =e^{\frac{i}{2} \pi N[(N-1) \beta+2 \gamma]} \Gamma\left(1-\frac{\beta}{2}\right)^{N} \prod_{l=1}^{N} \frac{\Gamma\left(1+\alpha+\frac{\beta}{2}(l-1)\right)}{\Gamma\left(1-\frac{\beta l}{2}\right) \Gamma\left(1-\gamma-\frac{\beta}{2}(l-1)\right) \Gamma\left(1+\alpha+\gamma+N \beta-\frac{\beta}{2}(l+1)\right)}
\end{aligned}
$$

If we expand (50) to $O\left(j^{2}\right)$ and use the identities

$$
\begin{aligned}
& \left(z_{1}+z_{2}\right)\left(z_{1}^{-1}+z_{2}^{-1}\right)=\frac{\left(z_{1}+z_{2}\right)^{2}}{z_{1} z_{2}} \\
& \left(z_{1}+z_{2}\right)=z_{1} z_{2}+1-\left(1-z_{1}\right)\left(1-z_{2}\right)
\end{aligned}
$$

we find $\left(I(n, m) \equiv I_{N}(n, \hbar+m,-2 \hbar)\right)$

$$
\begin{aligned}
\tilde{f}_{-11}^{(2)} & =1+\frac{\hbar}{I_{2}(0,0)}\left(2 I_{2}(0,0)+I_{2}(2,-1)+I_{2}(0,-1)+I_{2}(0,1)-2 I_{2}(1,-1)-2 I_{2}(1,0)\right) \\
& =\frac{1+\hbar}{1-\hbar}
\end{aligned}
$$

Similarly we can compute

$$
\tilde{f}_{-22}^{(2)}=\frac{(1+\hbar)\left(2-3 \hbar+2 \hbar^{2}\right)}{2(1-\hbar)(2-\hbar)}
$$

etc, all in agreement with the exact results given in [4] evaluated at $p=2 i \hbar$.

Note that it is because of the identities (52) and their analogues for $n=2,3, \ldots$ that we can evaluate $Z[j]$ with the help of (51). For $N=3$ this is, unfortunately, not possible and we have not been able to evaluate the functional integral for $N>2$. However the evaluation of three and higher point functions is possible as long as $N=2$. As examples we present two three-point functions. For instance for $\tilde{f}_{-211}^{(3)}$ we use the identity

$$
\left(z_{1}+z_{2}\right)^{2}\left(z_{1}^{-2}+z_{2}^{-2}\right)=\frac{1}{z_{1}^{2} z_{2}^{2}}\left(z_{1}+z_{2}\right)^{4}-\frac{2}{z_{1} z_{2}}\left(z_{1}+z_{2}\right)^{2}
$$

and together with the second identity in (52) we can express the integrand in a form which allows the use of (51). We find

$$
\tilde{f}_{-211}^{(3)}=\frac{2 \hbar(\hbar+1)}{(1-\hbar)(2-\hbar)}
$$

and similarly

$$
\tilde{f}_{-321}^{(3)}=\frac{2 \hbar(1+\hbar)}{(1-\hbar)(2-\hbar)(3-\hbar)}
$$

both in agreement with the exact results (cf. [4]) evaluated at $p=2 i \hbar$. 
The above calculations are non-perturbative checks of the proposal that the $S$-matrix of Liouville theory is represented by the functional integral (40) beyond the semi-classical approximation. The limitation is that we were only able to perform this check for special values of the center-of-mass momentum $p$, namely $p=i \hbar$ and $p=2 i \hbar$, but the checks were to all orders in $\hbar$.

It would be desirable to prove directly that the functional integral solves the equation for $\tilde{\mathcal{S}}$. More specifically, if we define

$$
\langle O\rangle_{j}=\frac{1}{\mathcal{N}} \int D \varphi O e^{-\frac{1}{\hbar} \Sigma\left(\varphi_{n}(|n|+i p) \varphi_{-n}-\varphi_{n} j_{-n}\right)}
$$

and insert the functional integral representation for $\tilde{\mathcal{S}}$ into (24), we obtain the equation

$$
\begin{aligned}
-n \eta & \left\langle\frac{\partial S}{\partial \varphi_{n}} e^{-V}\right\rangle_{j}+n(\eta|n|+i p)\left\langle\varphi_{-n} e^{-V}\right\rangle_{j} \\
& -b \sum_{k, l} l\left\langle\frac{\partial S}{\partial \varphi_{-k}} \varphi_{l} e^{-V}\right\rangle_{j} \delta_{k+l+n}+\frac{1}{2} b \sum_{k, l}(\epsilon(k)+\epsilon(l))\left\langle\varphi_{k} \varphi_{l} e^{-V}\right\rangle_{j} \delta_{k+l+n}=0
\end{aligned}
$$

Here

$$
S=\frac{1}{2} \sum \varphi_{n}(|n|+i p) \varphi_{-n}+V \quad \text { with } \quad V=i p \sum_{r \geq 3} \frac{b^{r-2}}{r !} \int \frac{d x}{2 \pi} \varphi^{r}(x)
$$

The sums are over all integers as the zero modes of $\varphi$ is included.

We have not succeeded of verifying (59) in general, but at first order in $\hbar$ and $j$, this is straightforward to do. Care is required when one performs the sums: each term is finite but the first sum is an infinite sum (while the second is over a finite range, due to the sign function and the Kronecker delta constraint).

One is tempted to move the sums under the integral and impose the condition that the integrand vanishes, thus leading to an equation for $S$, which can be solved. But this would lead to an incorrect form for $S$ which explicitly contains $\eta$, contrary to (34) and (35).

To summarize, we have proposed a functional integral representation for the generating functional for the $S$-matrix in Liouville theory and have performed perturbative and non-perturbative checks. It is an interesting question whether the methods used here can be applied to other theories.

\section{Acknowledgments}

We thank Daniele Dorigoni for a useful discussion. The work of G.J. was supported by the joint grant of Volkswagen Foundation and SRNSF (Ref. 93562 \& \#04/48).

\section{References}

[1] J. Teschner, "Liouville theory revisited," Class. Quant. Grav. 18 (2001) R153 [hepth/0104158]

[2] Y. Nakayama, "Liouville field theory: A Decade after the revolution," Int. J. Mod. Phys. A 19 (2004) 2771 [hep-th/0402009] 
[3] Alexei Zamolodchikov and Alexander Zamolodchikov, "Lectures on Liouville Theory and Matrix Models", online pdf version at http://qft.itp.ac.ru/ZZ.pdf

[4] G. Jorjadze and S. Theisen, "On the S-matrix of Liouville theory," JHEP 02 (2021), 111 [arXiv:2011.06876 [hep-th]]

[5] G. Jorjadze and G. Weigt, "Poisson structure and Moyal quantization of the Liouville theory," Nucl. Phys. B 619 (2001) 232 [hep-th/0105306]

[6] T. L. Curtright and C. B. Thorn, "Conformally Invariant Quantization of the Liouville Theory,” Phys. Rev. Lett. 48 (1982) 1309 [erratum: Phys. Rev. Lett. 48 (1982) 1768];

E. Braaten, T. Curtright and C. B. Thorn, "An Exact Operator Solution of the Quantum Liouville Field Theory,” Annals Phys. 147 (1983) 365

[7] H. Dorn and H. J. Otto, "Two and three point functions in Liouville theory," Nucl. Phys. B 429 (1994) 375 [hep-th/9403141],

H. Dorn and H. J. Otto, "Remarks on the continuum formulation of noncritical strings," hep-th/9212004

[8] A. B. Zamolodchikov and A. B.Zamolodchikov, "Structure constants and conformal bootstrap in Liouville field theory,” Nucl. Phys. B 477 (1996) 577 [hep-th/9506136]

[9] J. L. Gervais and J. Schnittger, "Continuous spins in 2-D gravity: Chiral vertex operators and local fields," Nucl. Phys. B 431 (1994), 273-314 [arXiv:hep-th/9405136]

[10] V. S. Dotsenko and V. A. Fateev, "Four Point Correlation Functions and the Operator Algebra in the Two-Dimensional Conformal Invariant Theories with the Central Charge c < 1, Nucl. Phys. B 251 (1985), 691-734 